\title{
Fragility of the Fractional Josephson Effect in Time-Reversal-Invariant Topological Superconductors
}

\author{
Christina Knapp $\odot,{ }^{1,2, *}$ Aaron Chew $\odot,{ }^{1, *}$ and Jason Alicea $\oplus^{1,2}$ \\ ${ }^{1}$ Department of Physics and Institute for Quantum Information and Matter, California Institute of Technology, \\ Pasadena, California 91125, USA \\ ${ }^{2}$ Walter Burke Institute for Theoretical Physics, California Institute of Technology, Pasadena, California 91125, USA
}

(Received 3 July 2020; accepted 1 October 2020; published 12 November 2020)

\begin{abstract}
Time-reversal-invariant topological superconductor (TRITOPS) wires host Majorana Kramers pairs that have been predicted to mediate a fractional Josephson effect with $4 \pi$ periodicity in the superconducting phase difference. We explore the TRITOPS fractional Josephson effect in the presence of time-dependent "local mixing" perturbations that instantaneously preserve time-reversal symmetry. Specifically, we show that just as such couplings render braiding of Majorana Kramers pairs nonuniversal, the Josephson current becomes either aperiodic or $2 \pi$ periodic (depending on conditions that we quantify) unless the phase difference is swept sufficiently quickly. We further analyze topological superconductors with $\mathcal{T}^{2}=+1$ time-reversal symmetry and reveal a rich interplay between interactions and local mixing that can be experimentally probed in nanowire arrays.
\end{abstract}

DOI: 10.1103/PhysRevLett.125.207002

Introduction.-Topological superconducting wires hosting unpaired end Majorana zero modes (MZMs) [1-10] display remarkably rich phenomenology with applications for topological quantum computation [11-15]. Wellseparated MZMs span a set of degenerate ground states that are locally indistinguishable and hence define a faulttolerant qubit subspace. Braiding unpaired MZMs implements universal non-Abelian rotations within the ground state subspace-thereby generating fault-tolerant qubit gates. Under a "fusion" process, a pair of MZMs brought together in space hybridizes and yields a finite-energy fermionic state that can be either empty ( $I$ fusion channel) or filled ( $\psi$ fusion channel). Detecting which fusion channel emerges provides a means of qubit readout.

An elegant method of probing topological superconductivity and performing readout utilizes the so-called fractional Josephson effect [1,16-18]. In a conventional Josephson junction, Cooper-pair tunneling generates a current that is $2 \pi$ periodic in the superconducting phase difference across the barrier. A pair of MZMs fused across a topological Josephson junction mediates single-electron tunneling, resulting in an anomalous $4 \pi$-periodic fractional Josephson current whose sign correlates with the associated fusion channel. This period doubling intimately relates to non-Abelian braiding: Advancing the phase difference by $2 \pi$ has the same effect as fully braiding the MZM pair on one side of the junction (in turn swapping the fusion channel $I \leftrightarrow \psi$ for the hybridized MZMs). Two such braids are necessary to return the system to its initial state, corresponding to $4 \pi$ phase evolution.

When some time-reversal symmetry $\mathcal{T}$ is present, topological superconducting wires can host multiple
MZMs at each boundary. In particular, a time reversal invariant topological superconductor (TRITOPS) for which $\mathcal{T}^{2}=-1$ [19-46] hosts a Kramers pair of end MZMs that cannot hybridize provided $\mathcal{T}$ is preserved. MZM Kramers pairs in a TRITOPS wire accordingly generate a symmetryprotected ground state degeneracy consisting of locally distinguishable states - and thus furnish a qubit subspace with limited fault tolerance. Indeed, even time-dependent local perturbations that instantaneously preserve $\mathcal{T}$ can rotate the Majorana Kramers pair wave functions, generating a nonuniversal non-Abelian Berry phase [47]. As a result of this "local mixing," braiding MZM Kramers pairs generically produces nonuniversal rotations in the groundstate subspace [48].

Given these nonuniversalities, to what extent does a fractional Josephson effect survive in TRITOPS wires? This question turns out to be exceedingly subtle. On one hand, in a TRITOPS Josephson junction that preserves $\mathcal{T}$ at phase differences 0 and $\pi$, each subgap level is certainly $4 \pi$ periodic (Fig. 1), suggesting that a fractional Josephson effect appears as predicted in numerous works $[26,29,30,43,49-57]$. But on the other, the braiding and fractional Josephson connection noted earlier naively implies that nonuniversality of the former spells doom for the latter. There is, however, reason for optimism: The Josephson-junction energy levels become degenerate only at discrete phase differences, suggesting that timedependent local perturbations may play a less dramatic role compared to the braiding problem (for which degeneracy persists throughout the evolution).

Here we show that, when the superconducting phase winds adiabatically, local mixing indeed spoils the 


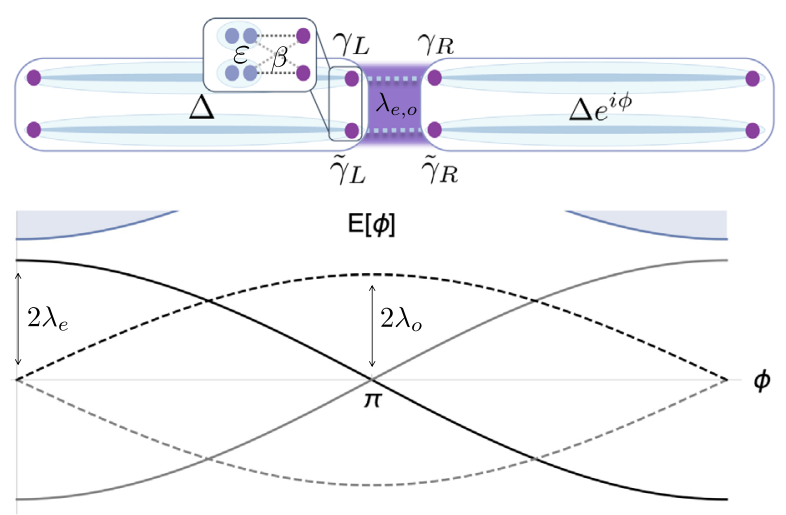

FIG. 1. Top panel: TRITOPS Josephson junction with each wire modeled by two time-reversed copies of a topological superconductor. Dotted lines indicate hybridization of Majorana Kramers pairs (dark purple) with magnitude $\lambda_{e, o}$ leading to Eq. (2). Inset: Local mixing arises when Majorana Kramers pairs undergo time-dependent coupling with amplitude $\beta$ to excited states (gray blue) at energy $\varepsilon$. Bottom panel: Many-body energy spectrum for a TRITOPS Josephson junction. Solid and dashed curves, respectively, correspond to even and odd fermionparity sectors.

fractional Josephson effect and yields either an aperiodic or $2 \pi$-periodic current-phase relation depending on localmixing timescales. This result holds even in an otherwise ideal situation for which effects known previously to destroy $4 \pi$ periodicity $[17,49,51,58-63]$ - e.g., explicit $\mathcal{T}$ breaking, overlap between distant MZMs, energy relaxation, and quasiparticle poisoning - are absent. By mapping the problem onto an effective model that features avoided crossings in the energy spectrum, we further demonstrate that $4 \pi$ periodicity is recovered when the phase difference evolves sufficiently quickly that local mixing remains benign. We extend our analysis to junctions of $\mathcal{T}^{2}=+1$ topological superconductors, which can be realized (approximately) with proximitized nanowire arrays [64]. Without interactions, local mixing can similarly spoil the fractional Josephson effect in a junction of $2 m$ wires when $m>1$. Interestingly, however, we find that interactions stabilize $4 \pi$ periodicity for any odd $m$ and $2 \pi$ periodicity when $m \bmod 4=0$. The nontrivial $m$ dependence reflects an interplay between local mixing and the $\mathbb{Z}_{8}$ classification of one-dimensional fermionic topological phases [65], and thus provides an experimental window into both phenomena. More generally, our analysis illustrates that $\mathcal{T}$-protected degeneracies are insufficient to protect adiabatic pumping cycles-including the $8 \pi$ Josephson effect predicted for proximitized quantum spin Hall systems [62,66-68].

Local mixing.-Let $\gamma_{1(2)}, \tilde{\gamma}_{1(2)}$ denote the MZM Kramers pair at the left (right) end of a TRITOPS wire. Time reversal sends

$$
\gamma_{j} \rightarrow s_{j} \tilde{\gamma}_{j}, \quad \tilde{\gamma}_{j} \rightarrow-s_{j} \gamma_{j}
$$

for convention-dependent signs $s_{j}= \pm 1$; note consistency with $\mathcal{T}^{2}=-1$. Bilinears hybridizing a given MZM Kramers pair are odd under $\mathcal{T}$ and thus forbidden.

Local, adiabatic time-dependent perturbations that instantaneously preserve $\mathcal{T}$ endow the MZM operators at each end with nontrivial time dependence. Such perturbations can result, e.g., from external manipulation or stochastic noise that couples the initial MZMs and bulk energy modes, as reviewed in the Supplemental Material [69]. Reference [47] showed that after the Hamiltonian completes a closed cycle in time $T$, the final state generically differs in a nonuniversal way from the initial state. The ground-state rotation resulting from this "local mixing" is implemented by the unitary matrix $U=\exp \left[\sum_{j=1,2} \theta_{j} \gamma_{j}(0) \tilde{\gamma}_{j}(0) / 2\right]$, with $\theta_{j}$ a local-mixing angle determined by evolution details. Local mixing accordingly spoils the topological protection of braiding MZM Kramers pairs [48].

TRITOPS Josephson junction.—Consider a TRITOPS Josephson junction (Fig. 1, top) with superconducting phase $\phi_{L}=0$ on the left and $\phi_{R}=\phi$ on the right. The Majorana Kramers pair $\gamma_{L}, \tilde{\gamma}_{L}$ at the left side of the junction hybridizes with the Majorana Kramers pair $\gamma_{R}, \tilde{\gamma}_{R}$ on the right side, mediating a supercurrent contribution that we wish to explore in the presence of local mixing. When $\phi=n \pi$ for $n \in \mathbb{Z}$ the junction preserves $\mathcal{T}$; at these values we adopt a convention where $\gamma_{L}, \tilde{\gamma}_{L}$ transform under $\mathcal{T}$ according to Eq. (1) with $s_{L}=1$, while $\gamma_{R}, \tilde{\gamma}_{R}$ transforms with $s_{R}=-(-1)^{n}$. A minimal time-independent junction Hamiltonian compatible with this symmetry reads

$$
\begin{aligned}
H_{\mathrm{JJ}}= & i \lambda_{e} \cos (\phi / 2)\left(\gamma_{L} \gamma_{R}+\tilde{\gamma}_{L} \tilde{\gamma}_{R}\right) \\
& +i \lambda_{o} \sin (\phi / 2)\left(\gamma_{L} \gamma_{R}-\tilde{\gamma}_{L} \tilde{\gamma}_{R}\right),
\end{aligned}
$$

where $\lambda_{e, o}$ are real-valued tunneling amplitudes [69]. In the even-parity sector $\left(i \gamma_{L} \gamma_{R}\right)\left(i \tilde{\gamma}_{L} \tilde{\gamma}_{R}\right)=+1$ only the first line survives, whereas in the odd-parity sector $\left(i \gamma_{L} \gamma_{R}\right)\left(i \tilde{\gamma}_{L} \tilde{\gamma}_{R}\right)=-1$ only the second line survives [72]. Figure 1, bottom panel, sketches the corresponding energyphase relation $E(\phi)$. Each subgap energy is $4 \pi$ periodic in $\phi$, and hence at this level of analysis the Josephson current $\langle I(\phi)\rangle=(2 e / \hbar)(d E / d \phi)$ is also $4 \pi$ periodic under adiabatic phase evolution.

Crucially, however, the TRITOPS fractional Josephson effect hinted at here does not constitute a robust adiabatic cycle. As proof of concept, suppose that we begin in the ground state of the even-parity sector (solid lines in Fig. 1, bottom), and then implement the following process: (i) adiabatically wind $\phi$ from 0 to $\pi$, yielding twofold Kramers degeneracy, (ii) turn on a local-mixing closed adiabatic subcycle, and (iii) adiabatically wind $\phi$ from $\pi$ to $2 \pi$. After stage (ii) local mixing rotates the system into a superposition of even-parity junction eigenstates via $U_{\mathrm{JJ}}=$ $\exp \left[\sum_{J=L, R} \theta_{J} \gamma_{J}(0) \tilde{\gamma}_{J}(0) / 2\right]$ for some nonuniversal $\theta_{L, R}$ mixing angles. Specifically, if $|-\rangle$ and $|+\rangle$ denote states 
that, respectively, evolve from the even-parity ground state and excited state at $\phi=\pi$, the system evolves to

$U_{\mathrm{JJ}}|-\rangle=\cos \left(\frac{\theta_{R}-\theta_{L}}{2}\right)|-\rangle+i \sin \left(\frac{\theta_{R}-\theta_{L}}{2}\right)|+\rangle$.

Repeated implementations of the closed adiabatic cycle above generically result in aperiodic unitary state evolution, signaling a breakdown of the TRITOPS fractional Josephson effect.

A more physically relevant scenario arises when local mixing and phase winding occur simultaneously. For an illustrative toy model, we incorporate a Kramers pair of Andreev bound states described by $f=\left(\gamma_{\varepsilon}^{\prime}+i \gamma_{\varepsilon}\right) / 2, \tilde{f}=$ $\left(\tilde{\gamma}_{\varepsilon}^{\prime}+i \tilde{\gamma}_{\varepsilon}\right) / 2$ and supplement Eq. (2) with [47]

$$
\begin{aligned}
& \delta H(t)=\frac{\varepsilon}{2}\left[-i\left(\gamma_{\varepsilon} \gamma_{\varepsilon}^{\prime}+\tilde{\gamma}_{\varepsilon} \tilde{\gamma}_{\varepsilon}^{\prime}\right)+2\right] \\
& \quad+i \frac{\beta}{2}\left[\cos \alpha(t)\left(\gamma_{L} \gamma_{\varepsilon}+\tilde{\gamma}_{L} \tilde{\gamma}_{\varepsilon}\right)+\sin \alpha(t)\left(\gamma_{L} \tilde{\gamma}_{\varepsilon}-\tilde{\gamma}_{L} \gamma_{\varepsilon}\right)\right] .
\end{aligned}
$$

The Andreev bound states exhibit an energy gap $\varepsilon$ encoded by the first line and, for simplicity, couple only to the Majorana Kramers pair on the left side of the junction via the second line; all terms instantaneously preserve $\mathcal{T}$. (Coupling the Andreev states also to the right side of the junction will not qualitatively change our conclusions.) Time dependence in $\alpha$ generically arises from noise.

We take $\varepsilon$ to be the largest energy scale and project onto the even-parity subspace $\gamma_{L} \gamma_{R} \tilde{\gamma}_{L} \tilde{\gamma}_{R} \gamma_{\varepsilon} \gamma_{\varepsilon^{\prime}} \tilde{\gamma}_{\varepsilon} \tilde{\gamma}_{\varepsilon}^{\prime}=+1$; working in the odd-parity subspace shifts the level crossings to $\phi=0$ but otherwise yields the same physics. In this formulation, the Hamiltonian $H(t)=H_{\mathrm{JJ}}+\delta H(t)$ supports two "low-energy"instantaneous eigenstatesdenoted $\left|\psi_{1}(t)\right\rangle$ and $\left|\psi_{2}(t)\right\rangle$-separated from the next lowest instantaneous eigenstates by an excess energy $\sim \mathcal{E}$. We further assume that $\alpha(t)$ varies slowly in time, i.e., $\beta \dot{\alpha}(t) \ll \varepsilon^{2}$, so that transitions between the low- and highenergy states are negligible. Solutions to Schrödinger's equation then approximately take the form $|\Phi(t)\rangle=$ $v_{1}(t)\left|\psi_{1}(t)\right\rangle+v_{2}(t)\left|\psi_{2}(t)\right\rangle$; the coefficients satisfy the equation of motion $i \partial_{t} \vec{v}=\left[H_{\text {inst }}(t)+H_{B}(t)\right] \vec{v} \equiv$ $H_{\text {eff }}(t) \vec{v}$, with $H_{\text {inst }}$ a diagonal matrix populated by the instantaneous energies and $H_{B, i j}=-\left\langle\psi_{i}(t)\left|i \partial_{t}\right| \psi_{j}(t)\right\rangle$ a Berry-phase term. Retaining terms up to $\mathcal{O}\left(\varepsilon^{-2}\right)$ (except an unimportant term proportional to identity), we explicitly find

$$
H_{\mathrm{eff}}(t)=2 \lambda_{e}^{\prime} \cos [\phi(t) / 2] \sigma_{z}-\frac{1}{2} \dot{\theta}(t) \sigma_{y},
$$

where $\lambda_{e}^{\prime}=\lambda_{e}\left[1-\beta^{2} /\left(2 \varepsilon^{2}\right)\right]$ is a renormalized tunneling amplitude, $\dot{\theta}(t)=-\dot{\alpha}(t) \beta^{2} / \varepsilon^{2}$, and the Pauli matrices now refer to the basis of instantaneous eigenstates $\left|\psi_{j}(t)\right\rangle$. When

\section{Transition Probability}

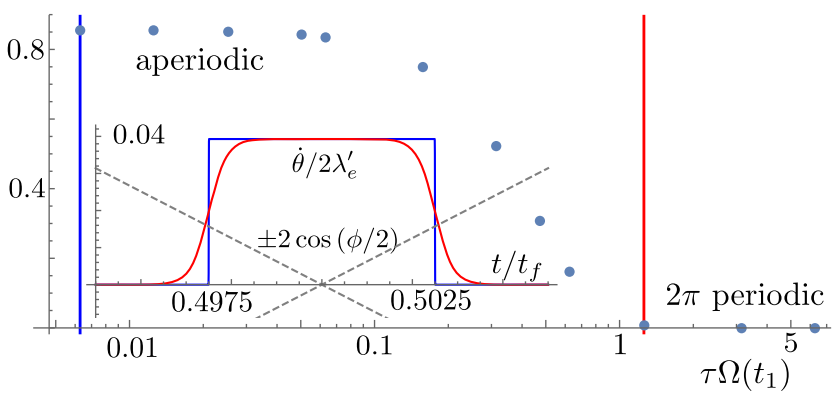

FIG. 2. Transition probability after evolution by $H_{\text {eff }}(t)$ from $t=0$ to $t_{f}$ for $\alpha(t)=2 \pi n\left[f(t)-f\left(t_{i}\right)\right] /\left[f\left(t_{f}\right)-f\left(t_{i}\right)\right]$ with $f(t)=\log \left[\left(1+e^{\left(t-t_{1}\right) / \tau}\right) /\left(1+e^{\left(t-t_{2}\right) / \tau}\right)\right]$ and $\phi(t)=2 \pi t / t_{f}$. Parameters are $\lambda_{e}=\varepsilon / 80, \quad \beta=\varepsilon / 40, \quad t_{f}=2 \times 10^{7} \varepsilon^{-1}$, $t_{1 / 2}=t_{f} / 2 \pm 0.0025 t_{f}$, and $n=25 \times 10^{3}$. The choice of $\alpha(t)$ yields steplike jumps in $\dot{\theta}(t)$ over a timescale $\tau$, as shown in the inset. In the main plot's horizontal axis, $\Omega\left(t_{1}\right)$ denotes the magnitude of the instantaneous energy at the time of the jumps, $\Omega\left(t_{1}\right)=\sqrt{4 \lambda_{e}^{\prime 2} \cos ^{2}\left[\phi\left(t_{1}\right) / 2\right]+\dot{\theta}\left(t_{1}\right)^{2} / 4}$. "Slow" jumps $\left[\tau \Omega\left(t_{1}\right) \gtrsim 1\right]$ yield nearly zero transition probability, signifying a $2 \pi$-periodic Josephson effect. "Fast" jumps $\left[\tau \Omega\left(t_{1}\right) \lesssim 1\right]$, however, generate aperiodicity — even for arbitrarily slow $\phi(t)$. Inset: Off-diagonal element of $H_{\text {eff }}(t) / \lambda_{e}^{\prime}$ near $\phi=\pi$ for $\tau \Omega\left(t_{1}\right) \approx$ 1.257 (red) and $\tau \Omega\left(t_{1}\right) \approx 0.00629$ (blue), along with diagonal elements (gray). Blue and red curves correspond to the $\tau \Omega\left(t_{1}\right)$ values indicated by the respective vertical lines in the main figure.

$\lambda_{e}^{\prime}=0$, the time-evolution operator $U=e^{-i \int_{0}^{T} H_{\text {eff }}(t)} \equiv$ $e^{i(\theta / 2) \sigma_{y}}$ implements local mixing among the degenerate junction states with mixing angle $\theta=\int_{0}^{T} d t \dot{\theta}(t)$, in agreement with Refs. [47,73] at $\beta \ll \varepsilon$. More interestingly, with $\lambda_{e}^{\prime} \neq 0$, the $\sigma_{y}$ term responsible for local mixing effectively couples the bound states related by time-reversal symmetry - even though the crossings in Fig. 1 are protected.

To analyze the Josephson effect described by Eq. (5), we first consider $\dot{\phi}$ and $\dot{\theta}$ approximately constant. Treating local mixing as a small perturbation away from the timereversal-invariant point, we expand $H_{\text {eff }}(t)$ near $\phi=\pi$ to obtain a standard Landau-Zener Hamiltonian. The transition probability between instantaneous $H_{\text {eff }}(t)$ eigenstates monotonically increases as $x=\dot{\theta}^{2} / \lambda_{e}^{\prime} \dot{\phi}$ decreases. At $x \gg 1$, the adiabatic criterion is satisfied; here a system initialized into the instantaneous ground state at $\phi=0$ evolves into the instantaneous ground state at $\phi=2 \pi$, yielding a $2 \pi$-periodic current-phase relation. For $x \ll 1$ "fast" phase winding instead overwhelms local mixing, and a $4 \pi$-periodic fractional Josephson effect emerges.

Next we examine a "quench" that more closely resembles the proof-of-concept picture considered earlier: As sketched in the inset of Fig. 2, during an interval at which $\phi \approx \pi, \dot{\theta}(t)$ jumps from zero to a finite value over a timescale $\tau$ and then similarly decays back to zero according to a smoothed-out rectangular pulse turned on 


\begin{tabular}{|c|c|c|c|c|}
\hline$\bullet$ & $\gamma_{L 1}$ & $\gamma_{R}$ & 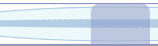 & 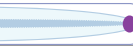 \\
\hline$\bullet$ & $\gamma_{L 2}$ & $\gamma_{R}$ & $\Delta e^{i \phi}$ & $\bullet$ \\
\hline & $\vdots$ & & $\vdots$ & \\
\hline$\bullet$ & $\gamma_{L m \bullet}$ & $\gamma_{R}$ & 2 & 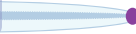 \\
\hline$m \bmod 4$ & non-interactir & $(\phi)$ & interacting & $\operatorname{g} I(\phi)$ \\
\hline 1 & $4 \pi$ & & $4 \pi$ & \\
\hline 2 & $2 \pi, 4 \pi$, or ap & dic & $2 \pi, 4 \pi$, or ap & periodic \\
\hline 3 & $4 \pi$ or aperi & & $4 \pi$ & \\
\hline 4 & $2 \pi, 4 \pi$, or ap & dic & $2 \pi$ & \\
\hline
\end{tabular}

FIG. 3. Josephson junction between two sets of $m$ proximitized nanowires that each (approximately) obey $\mathcal{T}^{2}=+1$ time-reversal symmetry [64]. Table: Summary of the periodicity for the current-phase relation in the presence of local mixing, both in the noninteracting limit and with interactions. Multiple values are given when the result depends on local-mixing details.

and off at times $t_{1}$ and $t_{2}$, respectively. This choice of $\theta(t)$ is motivated by the understanding that local mixing only plays a significant role near the time-reversal-invariant point, and that both its magnitude and time evolution affect the transition probability. Figure 2, main panel, depicts the numerically obtained transition probability as a function of $\tau \Omega\left(t_{1}\right)$. Here $2 \Omega\left(t_{1}\right)$ is the instantaneous gap evaluated at the jump and defines a natural energy for normalizing the timescale $\tau$; see caption for full parameters [69]. For "large" $\tau \Omega\left(t_{1}\right)$ [smooth $\theta(t)$ evolution] a localmixing-induced $2 \pi$-periodic Josephson effect arises, while as $\tau \Omega\left(t_{1}\right)$ decreases [ $\theta(t)$ approaches quench dynamics] the transition probability becomes appreciable and eventually plateaus-indicating an aperiodic current-phase relation.

Thus far we have focused on unitary time evolution. We now note that continuously measuring the current can stabilize $4 \pi$ periodicity through the quantum Zeno effect. Although current eigenstates correspond to energy eigenstates, the current is most distinguishable when the energies are degenerate; hence measurement backaction competes against local mixing. If the measurement projects onto a current eigenstate faster than the timescale of local mixing, the fractional Josephson effect survives (up to processes not considered here).

$\mathcal{T}^{2}=+1$ Josephson junction.-Topological superconductors with $\mathcal{T}^{2}=+1$ time-reversal symmetry can support an arbitrary number $m$ of MZMs at each end in the noninteracting limit, but only $m$ mod 8 with interactions [65]. As a physical realization, we envision an array of $m$ proximitized semiconductor nanowires in a magnetic field $[10,74-76]$, for which the minimal low-energy Hamiltonian preserves $\mathcal{T}^{2}=+1$ symmetry [64]. Figure 3, top, sketches a Josephson junction assembled from such arrays. Our goal is to explore the impact of local mixing and interactions on the Josephson effect.

Consider first the noninteracting limit. The junction hosts MZMs $\gamma_{L 1, \ldots, m}$ from the left end and $\gamma_{R 1, \ldots, m}$ from the right, which hybridize via

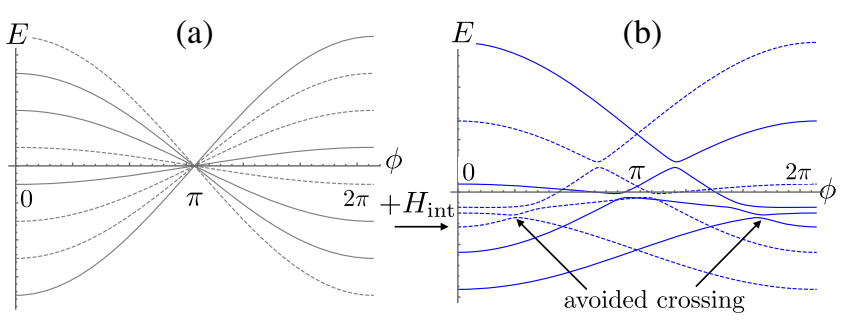

FIG. 4. (a) Noninteracting many-body spectrum versus $\phi$ for the Josephson junction in Fig. 3 in the $m=3$ case. Solid and dashed lines respectively denote even- and odd-fermion-parity states. Crossings necessarily occur at $\phi=\pi$ due to $\mathcal{T}^{2}=+1$ symmetry, and local mixing generically rotates among equalparity degenerate states. (b) Many-body spectrum with interactions - which shift the locations of the crossings away from the time-reversal-invariant point, where they are no longer protected. Interaction-induced avoided crossings between same-parity states protect the fractional Josephson effect against local mixing.

$$
H_{\mathrm{JJ}}^{\mathcal{T}^{2}=+1}=\sum_{j, k} i \lambda_{j k} \cos (\phi / 2) \gamma_{L j} \gamma_{R k}
$$

Time-reversal symmetry persists at $\phi=n \pi(n \in \mathbb{Z})$; moreover, at $\phi=\pi$ the hybridization vanishes, yielding $2 m$ MZMs at the junction. As for the $\mathcal{T}^{2}=-1$ case, each energy described by Eq. (6) is $4 \pi$ periodic in $\phi$, and hence a fractional Josephson effect exists at this level of analysis. Figure 4(a) illustrates the energies versus $\phi$ for $m=3$, with solid and dashed lines, respectively, denoting even- and odd-parity states.

To incorporate local mixing, observe that $\delta H(t)$ in Eq. (4) also preserves $\mathcal{T}^{2}=+1$ symmetry (with $\gamma_{L}, \tilde{\gamma}_{L}, \gamma_{\varepsilon}^{\prime}, \tilde{\gamma}_{\varepsilon}^{\prime} \rightarrow \gamma_{L}, \tilde{\gamma}_{L}, \gamma_{\varepsilon}^{\prime}, \tilde{\gamma}_{\varepsilon}^{\prime}$ and $\left.\gamma_{\varepsilon}, \tilde{\gamma}_{\varepsilon} \rightarrow-\gamma_{\varepsilon},-\tilde{\gamma}_{\varepsilon}\right)$. Thus one can immediately construct a local-mixing Hamiltonian for the $\mathcal{T}^{2}=+1$ problem by replacing $\gamma_{L} \rightarrow \gamma_{L j}, \tilde{\gamma}_{L} \rightarrow \gamma_{L k}$ in $\delta H(t)$ and summing over $j, k$ pairs. The net effect is that local mixing can once again nonuniversally rotate the system among same-parity Hamiltonian eigenstates that are degenerate at $\phi=\pi$. For any $m>1$ this degeneracy is nontrivial, implying that local mixing spoils the fractional Josephson effect unless the phase is swept sufficiently rapidly. Interestingly, for odd $m>1$ local mixing can never generate $2 \pi$ periodicity since the junction parity switches upon sweeping $\phi$ by $2 \pi$; see, e.g., Fig. 4(a).

Symmetry-preserving interactions, which we now turn on, substantially enrich this story. First, one only needs to consider $m$ mod 4. Indeed with $m=4$ the junction at $\phi=\pi$ hosts $8 \mathrm{MZMs}$ - whose degeneracy interactions completely obliterate [65], thus stabilizing $2 \pi$ periodicity. For $m \bmod 4=1$ a given fermion-parity sector has a unique ground state at $\phi=\pi$, so the fractional Josephson effect is immune to local mixing. The case $m \bmod 4=2$ essentially reduces to the TRITOPS Josephson junction already examined in great detail; at $\phi=\pi$ a twofold degeneracy in a given parity sector persists even with interactions, and local mixing accordingly generates $2 \pi$ periodicity, $4 \pi$ 
periodicity, or aperiodicity depending on details. Finally, for $m \bmod 4=3$ interactions shift crossings between sameparity states [recall Fig. 4(a)] away from $\phi=\pi$, where they become avoided crossings due to the absence of $\mathcal{T}$ symmetry; see Fig. 4(b). Interactions consequently protect the fractional Josephson effect against local mixing for adiabatic evolution. For nonadiabatic evolution, the timescale of local mixing should be compared to the combination of the interaction-induced energy gaps and the effective coupling between bound states mediated by local mixing. See the Table from Fig. 3 for a summary.

Discussion.-A very general implication of our study is that symmetry-protected degeneracies among locally distinguishable states do not necessarily suffice for generating robust nontrivial adiabatic cycles; examination of dynamics under generic conditions is additionally required. We have seen that $\mathcal{T}$-symmetric local mixing perturbations that render braiding nonuniversal in TRITOPS wires $[47,48]$ also preclude a well-defined adiabatic fractional Josephson effect. Similar results hold in junctions of $\mathcal{T}^{2}=+1$ topological superconductors, with the interesting addition that interactions in some cases immunize against local mixing. Our analysis extends previous works studying the fragility of time reversal symmetry protected effects $[47,48,77,78]$ to pumping cycles that only preserve the symmetry at discrete points in parameter space. Whether an analogous fate befalls cycles in systems with degeneracies protected by local unitary symmetries remains an interesting open question.

Our findings are relevant for experiments on both TRITOPS and nanowire-based Josephson junctions. Recent experiments investigating the Josephson effect in proximitized quantum spin Hall edges [79-81] observed signatures of $4 \pi$ and $2 \pi$ periodicity, whereas theory predicts an $8 \pi$-periodic Josephson effect [62,66-68]. Subgap energy levels corresponding to the same fermion parity are predicted to have a Kramers degeneracy at integer multiples of $\phi=\pi$; thus, local mixing could induce transitions at these time-reversal-invariant points. Additionally, the degree of $\mathcal{T}^{2}=+1$ symmetry breaking in Majorana nanowires has important implications for topological quantum computing with MZMs [13-15]. Projective MZM parity measurements proposed in Ref. [13] rely on pairs of MZMs hybridizing through adjacent quantum dots. Estimating the magnitude of symmetry breaking, e.g., by observing the timescale of $\dot{\phi}(t)$ for which the junction in Fig. 3 recovers a periodic Josephson effect, would bound the visibility of these measurements.

We are grateful to Arbel Haim, Torsten Karzig, and Yang Peng for illuminating discussions. We also thank Dima Pikulin and Charlie Marcus for conversations that stimulated this research. This work was supported by the Army Research Office under Grant No. W911NF-17-1-0323; the National Science Foundation through Grant No. DMR1723367; the Caltech Institute for Quantum Information and Matter, an NSF Physics Frontiers Center with support of the Gordon and Betty Moore Foundation through Grant No. GBMF1250; the Walter Burke Institute for Theoretical Physics at Caltech; and the Gordon and Betty Moore Foundations EPiQS Initiative, Grant No. GBMF8682.

*These authors contributed equally to this work.

[1] A. Y. Kitaev, Phys. Usp. 44, 131 (2001).

[2] C. Beenakker, Annu. Rev. Condens. Matter Phys. 4, 113 (2013).

[3] J. Alicea, Rep. Prog. Phys. 75, 076501 (2012).

[4] M. Leijnse and K. Flensberg, Semicond. Sci. Technol. 27, 124003 (2012).

[5] M. Sato and S. Fujimoto, J. Phys. Soc. Jpn. 85, 072001 (2016).

[6] T. D. Stanescu and S. Tewari, J. Phys. Condens. Matter 25, 233201 (2013).

[7] S. R. Elliott and M. Franz, Rev. Mod. Phys. 87, 137 (2015).

[8] S. D. Sarma, M. Freedman, and C. Nayak, npj Quantum Inf. 1, 15001 (2015).

[9] R. Aguado, Riv. Nuovo Cimento Soc. Ital. Fis. 40, 523 (2017).

[10] R. M. Lutchyn, E. P. a. M. Bakkers, L. P. Kouwenhoven, P. Krogstrup, C. M. Marcus, and Y. Oreg, Nat. Rev. Mater. 3, 52 (2018).

[11] A. Y. Kitaev, Ann. Phys. (Amsterdam) 303, 2 (2003).

[12] C. Nayak, S. H. Simon, A. Stern, M. Freedman, and S. Das Sarma, Rev. Mod. Phys. 80, 1083 (2008).

[13] T. Karzig, C. Knapp, R. M. Lutchyn, P. Bonderson, M. B. Hastings, C. Nayak, J. Alicea, K. Flensberg, S. Plugge, Y. Oreg et al., Phys. Rev. B 95, 235305 (2017).

[14] S. Plugge, A. Rasmussen, R. Egger, and K. Flensberg, New J. Phys. 19, 012001 (2017).

[15] S. Vijay and L. Fu, Phys. Rev. B 94, 235446 (2016).

[16] L. Fu and C. L. Kane, Phys. Rev. Lett. 100, 096407 (2008).

[17] L. Fu and C. L. Kane, Phys. Rev. B 79, 161408 (2009).

[18] H.-J. Kwon, K. Sengupta, and V. M. Yakovenko, Eur. Phys. J. B 37, 349 (2003).

[19] X.-L. Qi, T. L. Hughes, S. Raghu, and S.-C. Zhang, Phys. Rev. Lett. 102, 187001 (2009).

[20] L. Fu and E. Berg, Phys. Rev. Lett. 105, 097001 (2010).

[21] L. Santos, T. Neupert, C. Chamon, and C. Mudry, Phys. Rev. B 81, 184502 (2010).

[22] S. Deng, L. Viola, and G. Ortiz, Phys. Rev. Lett. 108, 036803 (2012).

[23] S. Deng, L. Viola, and G. Ortiz, Phys. Rev. Lett. 108, 036803 (2012).

[24] S. Nakosai, Y. Tanaka, and N. Nagaosa, Phys. Rev. Lett. 108, 147003 (2012).

[25] C. L. M. Wong and K. T. Law, Phys. Rev. B 86, 184516 (2012).

[26] A. Keselman, L. Fu, A. Stern, and E. Berg, Phys. Rev. Lett. 111, 116402 (2013).

[27] E. Dumitrescu and S. Tewari, Phys. Rev. B 88, 220505(R) (2013).

[28] S. Nakosai, J. C. Budich, Y. Tanaka, B. Trauzettel, and N. Nagaosa, Phys. Rev. Lett. 110, 117002 (2013). 
[29] F. Zhang, C. L. Kane, and E. J. Mele, Phys. Rev. Lett. 111, 056402 (2013).

[30] X.-J. Liu, C. L. M. Wong, and K. T. Law, Phys. Rev. X 4, 021018 (2014).

[31] K. Sun, C.-K. Chiu, H.-H. Hung, and J. Wu, Phys. Rev. B 89, 104519 (2014).

[32] A. Haim, A. Keselman, E. Berg, and Y. Oreg, Phys. Rev. B 89, 220504(R) (2014).

[33] J. Klinovaja, A. Yacoby, and D. Loss, Phys. Rev. B 90, 155447 (2014).

[34] C. Schrade, A. A. Zyuzin, J. Klinovaja, and D. Loss, Phys. Rev. Lett. 115, 237001 (2015).

[35] D. I. Pikulin, Y. Komijani, and I. Affleck, Phys. Rev. B 93, 205430 (2016).

[36] A. Haim, K. Wölms, E. Berg, Y. Oreg, and K. Flensberg, Phys. Rev. B 94, 115124 (2016).

[37] H. Ebisu, B. Lu, J. Klinovaja, and Y. Tanaka, Prog. Theor. Exp. Phys. 2016, $083 \mathrm{I} 01$ (2016).

[38] Y. Kim, D. E. Liu, E. Gaidamauskas, J. Paaske, K. Flensberg, and R. M. Lutchyn, Phys. Rev. B 94, 075439 (2016).

[39] J. Li, W. Pan, B. A. Bernevig, and R. M. Lutchyn, Phys. Rev. Lett. 117, 046804 (2016).

[40] A. Alase, E. Cobanera, G. Ortiz, and L. Viola, Phys. Rev. Lett. 117, 076804 (2016).

[41] F. Parhizgar and A. M. Black-Schaffer, Sci. Rep. 7, 9817 (2017).

[42] C. Reeg, C. Schrade, J. Klinovaja, and D. Loss, Phys. Rev. B 96, 161407(R) (2017).

[43] A. Haim and Y. Oreg, Phys. Rep. 825, 1 (2018).

[44] A. A. Aligia and L. Arrachea, Phys. Rev. B 98, 174507 (2018).

[45] C. Schrade and L. Fu, Phys. Rev. Lett. 120, 267002 (2018).

[46] O. E. Casas, L. Arrachea, W. J. Herrera, and A. L. Yeyati, Phys. Rev. B 99, 161301 (2019).

[47] K. Wölms, A. Stern, and K. Flensberg, Phys. Rev. Lett. 113, 246401 (2014).

[48] K. Wölms, A. Stern, and K. Flensberg, Phys. Rev. B 93, 045417 (2016).

[49] S. B. Chung, J. Horowitz, and X.-L. Qi, Phys. Rev. B 88, 214514 (2013).

[50] F. Zhang and C. L. Kane, Phys. Rev. B 90, 020501(R) (2014).

[51] E. Mellars and B. Beri, Phys. Rev. B 94, 174508 (2016).

[52] W. Gong, Z. Gao, W. Shan, and G. Yi, Sci. Rep. 6, 23033 (2016).

[53] X. Liu, X. Li, D.-L. Deng, X.-J. Liu, and S. Das Sarma, Phys. Rev. B 94, 014511 (2016).

[54] A. Camjayi, L. Arrachea, A. Aligia, and F. von Oppen, Phys. Rev. Lett. 119, 046801 (2017).

[55] A. Alase, E. Cobanera, G. Ortiz, and L. Viola, Phys. Rev. B 96, 195133 (2017).

[56] E. Cobanera, A. Alase, G. Ortiz, and L. Viola, Phys. Rev. B 98, 245423 (2018).

[57] L. Arrachea, A. Camjayi, A. A. Aligia, and L. Gruñeiro, Phys. Rev. B 99, 085431 (2019).
[58] P. San-Jose, E. Prada, and R. Aguado, Phys. Rev. Lett. 108, 257001 (2012).

[59] D. I. Pikulin and Y. V. Nazarov, Phys. Rev. B 86, 140504(R) (2012).

[60] S.-P. Lee, K. Michaeli, J. Alicea, and A. Yacoby, Phys. Rev. Lett. 113, 197001 (2014).

[61] D. M. Badiane, L. I. Glazman, M. Houzet, and J. S. Meyer, C.R. Phys. 14, 840 (2013).

[62] Y. Peng, Y. Vinkler-Aviv, P. W. Brouwer, L. I. Glazman, and F. von Oppen, Phys. Rev. Lett. 117, 267001 (2016).

[63] D. Sticlet, J. D. Sau, and A. Akhmerov, Phys. Rev. B 98, 125124 (2018).

[64] S. Tewari and J. D. Sau, Phys. Rev. Lett. 109, 150408 (2012).

[65] L. Fidkowski and A. Kitaev, Phys. Rev. B 81, 134509 (2010).

[66] F. Zhang and C. L. Kane, Phys. Rev. Lett. 113, 036401 (2014).

[67] C. P. Orth, R. P. Tiwari, T. Meng, and T. L. Schmidt, Phys. Rev. B 91, 081406 (2015).

[68] H.-Y. Hui and J. D. Sau, Phys. Rev. B 95, 014505 (2017).

[69] See Supplemental Material at http://link.aps.org/ supplemental/10.1103/PhysRevLett.125.207002 for additional details about the effective Hamiltonian, local mixing, and Majorana nanowire arrays, which includes Refs. [70,71].

[70] M. Born and V. Fock, Z. Phys. 51, 165 (1928).

[71] D. A. Lidar, A. T. Rezakhani, and A. Hamma, J. Math. Phys. (N.Y.) 50, 102106 (2009).

[72] Interactions generate a four-body term $\propto \gamma_{L} \gamma_{R} \tilde{\gamma}_{L} \tilde{\gamma}_{R}$ that can further split the even- and odd-parity sectors, but do not play an important role in this setup.

[73] Up to a factor of two error in their Eq. (8a).

[74] R. M. Lutchyn, J. D. Sau, and S. Das Sarma, Phys. Rev. Lett. 105, 077001 (2010).

[75] Y. Oreg, G. Refael, and F. von Oppen, Phys. Rev. Lett. 105, 177002 (2010).

[76] S. Vaitieknas, G. W. Winkler, B. van Heck, T. Karzig, M.-T. Deng, K. Flensberg, L. I. Glazman, C. Nayak, P. Krogstrup, R. M. Lutchyn et al., Science 367, eaav3392 (2020).

[77] M. McGinley and N. R. Cooper, Phys. Rev. B 99, 075148 (2019).

[78] M. McGinley and N. R. Cooper, Nat. Phys. (2020) https:// doi.org/10.1038/s41567-020-0956-Z.

[79] E. Bocquillon, R. S. Deacon, J. Wiedenmann, P. Leubner, T. M. Klapwijk, C. Brne, K. Ishibashi, H. Buhmann, and L. W. Molenkamp, Nat. Nanotechnol. 12, 137 (2016).

[80] R. S. Deacon, J. Widenmann, E. Bocquillon, F. Dominguez, T. Klapwijk, P. Leubner, C. Brune, E. M. Hankiewicz, S. Tarucha, and K. Ishibashi, Phys. Rev. X 7, 021011 (2017).

[81] E. Bocquillon, J. Wiedenmann, R.S. Deacon, T. M. Klapwijk, H. Buhmann, and L. W. Molenkamp, Topological Matter, Springer Series in Solid-State Sciences 190 (Springer, New York, 2018), pp. 115-148. 